\title{
OPACs, caminos y desafíos 2.0: hacia la convergencia de medios y saberes.
}

\author{
Alicia García de León
}

\section{Gabriela Piñeyro Bascou}

\section{Resumen}

Este artículo describe los orígenes y la evolución histórica de los denominados catálogos en línea de Acceso Público (OPAC) centrado en los y desafíos del universo 2.0 y en la transformación de la relación de los usuarios con la información. Identifica los factores que incidieron en esa evolución, presentando las transformaciones que generan tanto la multiplicación de los soportes, como el impacto de la adopción masiva de las TIC.

Describe el universo 2.0 como un espacio de convergencia de herramientas, recursos y medios que interactúan y lo considera la causa central de su transformación que nace de de los requerimientos de los usuarios.

Expresa la necesidad de una reconversión actitudinal y emocional por parte de los profesionales de información y las bibliotecas, en el entendido de que la riqueza de la interacción transforma la vez a la biblioteca desde afuera hacia adentro.

\section{Palabras claves}

OPAC, Catalogos, OPAC 2.0, Web 2.0, Biblioteca 2.0, Interfaz, Biblioteca, Ergonomia, OPACS

\section{Roads and challenges 2.0 : toward the convergence of media and knowledge.}

\section{Abstract}

This paper describes the origins and the historical evolution of the so-called on-line catalogs of Public Access (OPAC) and focused on the challenges of the universe 2.0 and in the transformation of the relationship between users with the information. Identifies the factors that influenced this evolution, presenting the transformations that produce both the multiplication of the media, such as the impact of the mass adoption of ICTS. Describes the universe 2.0 as a space of convergence of tools, resources, and means that interact and considers it the central cause of the transformation that is born of the requirements of the users. Expresses the need for a conversion by attitudinal and emotional part of the information professionals and libraries, on the understanding that the richness of interaction transforms both to the library from the outside inside.

\section{Key Words}

OPAC, Catalogs Opac 2.0, Web 2.0, 2.0 Library, Interface, Library, Ergonomics

http://dx.doi.org/10.5209/rev_CDMU.2013.v24.46281 


\section{EN EL PRINCIPIO ERA EL CATÁLOGO}

Los catálogos bibliográficos, más allá de formas y soportes, nacen como herramientas de registro de documentos. Buscan identificar las obras, consignar sus rasgos distintivos y permitir su localización. Registran entre otros datos: autor o autores, título, volumen y ubicación.

Eran originalmente herramientas pensadas para el uso interno de las organizaciones, fueran estos monasterios, entidades educativas o de otra naturaleza y para ser manejadas por los administradores directos de los recursos básicamente los bibliotecarios.

Concebidos para ser leídos por el ojo humanos, su función central era identificar los documentos existentes. La exhaustividad y la fidelidad en el registro eran claves, el usuario, el acceso y la difusión no eran variables a considerar.

Originalmente listados en forma de rollos o libros, pasaron a convertirse en conjuntos de unidades móviles en soporte papel: las fichas normalizadas.

En estas etapas surgen requerimientos que llevan al catálogo a recorrer dos caminos:

- la normalización de los contenidos de la descripción;

- la resolución del acceso a los documentos desde distintos puntos, como título, temas o serie.

Con el tiempo, la descripción bibliográfica se normaliza. Se realiza un gran esfuerzo de diseño centrado en el objetivo de encontrar aquel o aquellos algoritmos aplicables a cualquier documento y que dieran por resultado una descripción e identificación estandardizada.

Paralelamente, las unidades móviles en soporte papel (fichas) también se normalizan no sólo en sus dimensiones, sino en la forma de presentar los documentos descriptos.

Convergiendo, a lo largo del tiempo, ambos caminos interactúan:

Se concibe una descripción normalizada en atención a su materialización en fichas de papel legibles por el ojo humano y se piensa en el acceso a partir de otras fichas desplegadas en ese mismo catálogo.

Una descripción nuclear (ficha principal) se incorpora a un juego de fichas, creándose otras iguales o similares que permiten accesos al mismo objeto desde otros puntos. Esas otras fichas son Ilamadas por esa razón secundarias.

En suma: los objetos documentales existentes, son descriptos según un canon y es posible encontrarlos con los ojos humanos mediante la recorrida manual y material de juegos de fichas intercalados en un catálogo. Así materializados: los viejos listados en forma de rollo o libro dejan paso a ficheros con unidades móviles normalizadas en su tamaño, contenido y estructura. 




Figura 1. Catálogo en fichas que ha sido familiar a los usuarios de varias generaciones y al que todavía se asocia la biblioteca en el imaginario colectivo.

\section{EL CATÁLOGO CRECE, HAGAMOS FICHAS...}

Para 1950 el catalogo bibliográfico en fichas parecía ser un producto consolidado y visto como una herramienta apropiada; un punto de llegada.

Algunas décadas atrás, los usuarios habían empezado a acceder al catálogo en la biblioteca y a recorrer las fichas que los llevaban a los documentos. No eran estructuras creadas originalmente para los usuarios, sino fuentes de uso interno, pensadas para los bibliotecarios.

Así considerados, como una herramienta cristalizada y universal, los problemas del catálogo bibliográfico se centraban en su materialización y cobertura. Había que hacer fichas, descripciones exhaustivas y fieles y crear accesos secundarios, preferentemente el mayor número. La misión era crear un buen objeto: el catálogo tal y como estaba concebido entonces.

En la década del 60 , se inicia un largo proceso donde la automatización se involucra con los catálogos. La idea inicial fue construir el catálogo en fichas tal y como era de uso, pero a partir de un mecanismo automático. Esto es: una máquina que crea fichas y por lo tanto, sigue la normativa que regía su construcción de modo manual. Debía ser iguales, pero "creadas por la máquina", pero para ser legibles por el ojo humano.

Así, las primeras formas de automatización en los catálogos bibliográficos se orientaron inicialmente a la creación de fichas de catálogos gobernadas por la normativa establecida y condicionada por la creación manual. Se replicaban fichas y esencialmente una lógica previa. 
Aún no se percibía que, junto a construir esas fichas en cantidad y con rapidez, se estaba almacenando información que podía ser recorrida y manejada en sí misma.

\section{HACEMOS FICHAS. CONSIGNAMOS INFORMACIÓN, PODEMOS INTERACTUAR.}

Con la incorporación de la automatización a la creación de catálogos, crecientes volúmenes de datos asociados a objetos documentales se consignaron.

Como ya vimos, los nuevos mecanismos de registro estaban orientados a hacer fichas bibliográficas, y gobernados por la normativa que pautó la construcción de las fichas manuales. Poseían la lógica de recuperación que proviene del catálogo manual donde a un acceso principal, se le suman otras fichas que proveen los accesos llamados secundarios.

La normativa y la lógica del catálogo clásico eran la pauta. Seguían siendo herramientas pensadas para los bibliotecarios, aunque el usuario pudiera tomar contacto con ellas. Estaban orientadas básicamente al registro exhaustivo y fiel de grandes volúmenes de documentos. No eran considerados centrales aspectos como la interacción o la recuperación.

En este período nacen algunos listados de documentos en forma de libro que reproducen los contenidos registrados. También se usan soportes como el microfilme y la microficha. Todas estas formas se concebían como extensiones de la lógica predominante llevada a otro soporte.

Sin embargo, los archivos que contenían los registros destinados a hacer fichas o listados que las reproducían, empieza a horadar aquella férrea estructura tributaria de la ficha bibliográfica. Grandes cantidades de datos normalizados, tienen ahora posibles nuevos vínculos y caminos que dejan esa lógica atrás.

Paralelamente, y en base a la forma de producción y los nuevos soportes, fichas creadas automáticamente, listados, microfilmes y microfichas llegaban a más personas y permitían además una novel interacción con los usuarios. Es el germen.

\section{LOS CATÁLOGOS EN LÍNEA}

Los archivos informáticos creados para hacer fichas o listados que las reprodujeran, abren otros caminos y otros mecanismos de acceso a un mayor número de usuario. Sin embargo la descripción nacida en el universo manual y la lógica que subyace en ese universo permanece.

Nace el catálogo en línea de acceso público llamados en su forma inglesa Online Public Access Catalogue $(O P A C)$. Tributarios de su impronta, pautados por la visión que les dio origen, en el futuro contendrán siempre esa dualidad y serán escenario de una lucha, a veces silenciosa, a veces estridente:

- ¿Qué describir y cómo?

- ¿Para quién y para quienes?

- ¿Orientado a consignar o a encontrar?

- ¿ ¿Orientados a encontrar como el ojo humano o con la lógica de la recuperación automática? 
- ¿Qué caminos se presentan para interactuar con ellos?

\section{TODO ES DIFERENTE, ODRES TAMBIÉN HACEN AL VINO.}

EI OPAC (Online Public Access Catalogue) o Catálogo en Línea de Acceso Público (CAPEL) nace en los años 70 como una versión automatizada de acceso remoto al catálogo en fichas bibliográficas.

Su condición de herramienta de acceso público y en línea, los convierte en un escenario diferente.

Ya no puede solamente reproducir la estructura consolidada en el pasado sino que conlleva el germen de la interacción. Se desplaza el eje original vinculado al bibliotecario, a la atención a los requerimientos del usuario.

Estamos en presencia de lo que Hildreth ${ }^{1}$ identifica como la primera de las 3 generaciones OPACs: "La primera generación intenta reproducir los catálogos tradicionales, brinda accesos mínimos, carece de interactividad y de normalización".

Los OPAC llegan a los usuarios, pero el mundo al que llegan y el usuario que encuentran es esencialmente diferente. Lejos está aquel que consultaba el catálogo en fichas o que buscaba datos en un CD.

El acceso a los equipos informáticos se amplía progresivamente; la operación de los recursos se convierte cada día en más amigable (claridad en diseño, ambientes gráficos, ayudas); la red Internet se hace cada día más accesible. Los costos de los equipos disminuyen y nacen los equipos personales (PCs).

Las personas se vinculan directamente con la información, buscan, recorren, acceden a recursos remotos y pueden andar esos caminos desde cualquier lugar con conexión, por ejemplo su casa, y en cualquier horario y día. En una primera lectura, en un análisis superficial: la mediación quedó atrás y muy poco tardó en ser proclamada.

Ciertamente que muy lejos están las unidades de información de quedar fuera de la tarea de disponibilizar recursos. Pero, para cumplir su misión de brindar información diseñando caminos apropiados, deberán aceptar el desafío de una reconversión técnica, profesional y hasta emocional.

Hay que pensar distinto sin por ello abdicar de la misión y mucho menos renunciar a la calidad.

Los usuarios son diferentes:

- el usuario de siempre que se convierte en nuevo porque, siendo el mismo, cambió su vínculo con la información, se apropió de otra lógica y tiene otros requerimientos;

- quien llega al catálogo por primera vez en este marco de acceso universal, no tiene experiencia y

${ }^{1}$ Hildreth, Charles R. Pursuing the ideal: generations of online catalogs. En: Aveney, B. y B. Butler, eds. Online catalogs, online reference: converging trends. Chicago: ALA. p. 31-56. 
sus expectativas vienen de la cultura de la recuperación en la red. 


\section{DISEÑAR EL CAMINO, COMPRENDER EL CAMINO: LA INTERFAZ}

Durante muchos años la misión de quién construía catálogos, sean estos listados, juegos de fichas o bases de datos, era describir con rigor y exhaustividad documentos, describir el mayor número y sin errores $u$ omisiones. Se reunían datos, en su momento datos normalizados y se reunían de la mejor manera, siguiendo pautas y estándares. La tarea concluía en la creación de un conjunto de registros sistematizados y consolidados en un soporte. Existían, estaban y por lo tanto podían ser utilizados.

Pero ocurrió que cuando esos datos consolidados pasan a vincularse con los usuarios en forma creciente, directa, y potencialmente universal: los caminos para encontrar los datos e interactuar con ellos pasan a ser tan importantes como los datos en sí mismos.

Sostiene Mari Carmen Marcos Moraㄹ : "La manera que se elija para presentar al usuario final la información es tan importante como el contenido de esos datos, ya que la forma en que se la haga accesible en pantalla va a determinar que la información cumpla su finalidad de ser útil o por el contrario se quede almacenada sin más..."

Nacen entonces esfuerzos por comprender y aplicar técnicas de recuperación de la información, y aportes de los estudios efectuados sobre la interacción de los usuarios con los sistemas de información. Un punto clave en la optimización de ese vínculo es la interfaz.

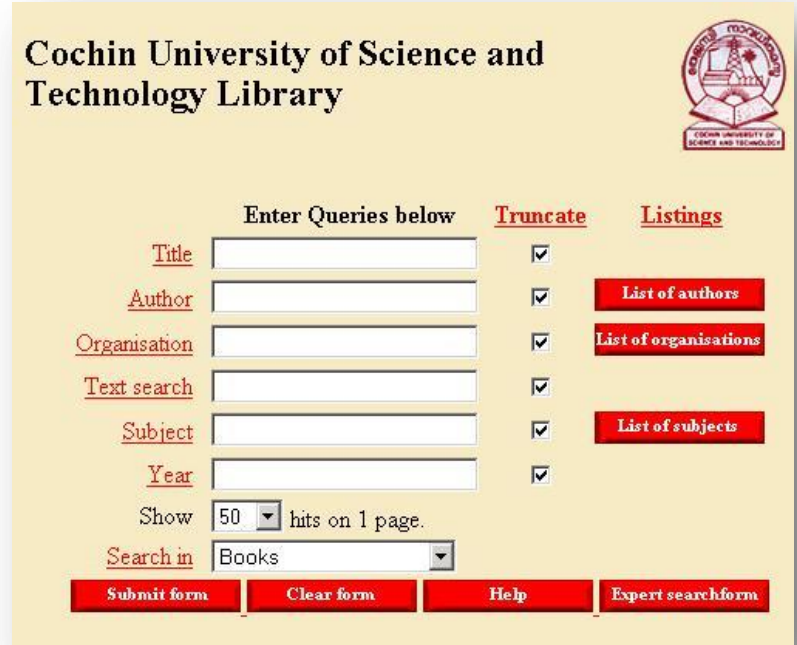

Figura 2. Las primeras interfaces presentaron al OPAC, replicando lógica de la recuperación manual.

\footnotetext{
${ }^{1}$ Marcos Mora, Mari-Carmen (2004). Pautas para el diseño y la evaluación de interfaces de usuario. En: Rovira, Cristòfol; Codina, Lluís; Marcos, Mari-Carmen; Palma, María del Valle. Información y documentación digital. Barcelona: IULA; Documenta Universitaria, 2004. ISBN 84-96367-09-6.
} 
Llamamos interfaz a la presentación en pantalla que un sistema informático ofrece al usuario para que este pueda interactuar con él. Es un ámbito destinado a la comunicación y a la interacción.

En el caso de las interfaces de los OPACs, estás deben "hacer justicia" al catálogo que presentan. Muchos son los catálogos que fracasan con una interfaz y que cambiándola se convierten en herramientas usadas, recomendadas y que fidelizan usuarios.

La regla clave de una interfaz es un diseño simple y claro, esencialmente intuitivo. En su diseño es clave el concepto de usabilidad. Con el aporte de muchas disciplinas como ergonomía cognitiva y la arquitectura de la información, los OPACs han evolucionado, mejorando su diseño y se han convertido en productos dirigidos a usuarios finales.

Así, el catálogo que nació como una herramienta de uso interno, y luego traspasó esos muros ampliándose al ámbito de un servicio y su clientela, se piensa hoy en una dimensión universal en el marco de Internet.

Existe un enorme trabajo de investigación, estudio y normalización en torno a la calidad de las interfaces para los OPACs. Corresponde señalar que muchos profesionales e instituciones comenzaron a pensar sus catálogos en torno al usuario, sus intereses y sus necesidades. Sin embargo, anticipando una brecha por demás previsible sostienen Rodríguez Yunta y Giménez Toledo ${ }^{1}$ : "Los profesionales de la documentación no pueden trabajar de espaldas a sus usuarios. Documentalistas y bibliotecarios deben superar cierto complejo de superioridad y desprecio hacia los usuarios..."

Estamos ante la llamada segunda generación de OPACs se proporcionan diversos puntos de acceso, posee una interfaz amigable y normaliza los formatos de descripción.

\section{PÚBLICO, DE ACCESO EN LÍNEA, EN LA WEB}

Sin duda el catálogo ha recorrido un largo camino, ha debido cambiar y aunque muchos cambios pueden identificarse como de magnitud o alcance, estamos en presencia de cambios de naturaleza.

No se trata de una herramienta vista por más, de un mayor número de documentos registrados o de más información integrada. Si bien esos cambios existen y han significado junto a otros un aporte a la herramienta, estamos en presencia de un cambio que hace a la naturaleza y no a la magnitud.

El usuario establece un nuevo vínculo con la información, con los contenidos y con los mediadores.

En una lectura ligera puede pensarse que un vínculo queda atrás, sin embargo se reformula y en ese marco los profesionales de la información son protagonistas desde otro lugar. Es el tiempo de junto a crear y registrar datos, diseñar los caminos y aportar en términos de capacitación para recorrerlos.

\footnotetext{
${ }^{1}$ Rodriguez Yunta, Luis; Giménez Toledo, Elea (2004). Lo que los usuarios piensan de las bases de datos bibliográficas y no se atreven a decir. ¿Es posible un diseño centrado en el usuario? Preprint de la primera versión de la Comunicación enviada a Infogestión. IX Jornadas Españolas de Documentación. Fesabid 14-15 abril 2005 Preprint entregado el 3 de noviembre de 2004.
} 
Los OPACs se presentan en sitios Web. Los sitios Web son estructuras de información. Las estructuras que contienen un OPAC, lo enmarcan o lo consideran un componente más de la oferta de recursos a los usuarios.

Ya en el año 2000 Michel Fingerhut ${ }^{1}$ abordaba el tema del sitio Web de la Biblioteca como un espacio más de esa biblioteca, parte de su planta física, digamos su planta lógica. En el 2002 María Victoria Játiva ${ }^{2}$ mencionaba el concepto de "OPAC Portal" a partir de la necesidad de "... Mejorar, ampliar e integrar recursos informativos".

Más tarde en el 2005 Tomás Saorín ${ }^{2}$ nos hablaba del "OPAC extendido" como un espacio donde se incorporan todas las prestaciones y funcionalidades de Internet. En el 2009 Nieves González ${ }^{7}$ habla de la "biblioteca expandida" y más recientemente se menciona el "OPAC Portal".

Se trata de lecturas sobre la necesidad de desarrollar y promover espacios integrados, espacios necesarios para el usuario y claves para la gestión de toda unidad de información. Ámbitos de convergencia de recursos, pensados para el trabajo, que reciben a los usuarios y les presentan documentos, herramientas y fuentes, así como asisten a la navegación e informan desde una única interfaz.

Si bien mencionamos visiones recientes de la reflexión académica, tempranamente los OPACs se presentaron tanto enriquecidos como asociados a una dimensión más vasta.

Debemos tener presente por ejemplo, los espacios Telnet en los orígenes de Internet, un protocolo que permite el acceso remoto emulando una terminal. Donde las prestaciones se sumaban. Telnet fue residencia de muchos OPACs hasta que migraron a espacios Web. Aún hoy día, muchas bibliotecas brindan este servicio.

\footnotetext{
${ }^{1}$ Fingerhut, Michel (2000). Le site Web de la bibliothèque considéré comme un espace. Bulletin des Bibliothéques de France, 45 (3), 78 - 82. Extraído el 21 de febrero de 2013 de: http://bbf.enssib.fr/consulter/10-finger.pdf
}

2 Játiva Miralles, Victoria (2002) Opac-portal: una nueva forma de ofrecer los recursos y servicios de la biblioteca. El Profesional de la Información, 11 (6), 442-453.

3 Saorín Pérez, Tomás (2006). Modelo conceptual para la automatización de bibliotecas en el contexto digital. Universidad de Murcia. [Tesis doctoral dirigida por José Vicente Rodríguez Muñoz].

$4 \quad$ González Fernández-Villavicencio, Nieves (2009). La biblioteca expandida en código abierto. Boletín de la Asociación Andaluza de Bibliotecarios, 24, (96-97). 


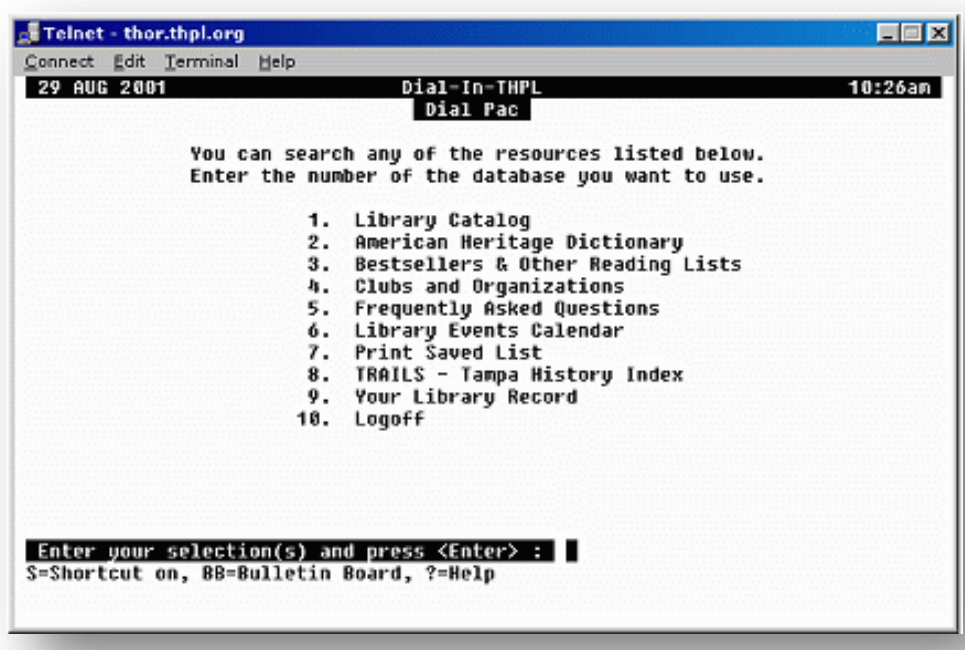

Figura 3. Mediante un sistema de menús, la biblioteca era presentada como un todo y el catálogo era un componente. Las primeras interfaces presentaron al OPAC, replicando lógica de la recuperación manual.

\section{Nuevos marcos, nuevos usuarios, nuevas demandas.}

La información se presenta ahora en nuevos espacios y soportes en el marco de las redes. Y su volumen crece exponencialmente. Las posibilidades de acceso y localización de documentos y fuentes se han multiplicado y sin embargo, la formación y asistencia a los usuarios, sean estos experimentados en el manejo de recursos, o noveles atraídos por las capacidades tecnológicas, no ha avanzado al mismo ritmo.

Ha existido cierta demora en identificar las dificultades y la necesidad de perfeccionar la comunicación del usuario con las herramientas y recursos. Durante mucho tiempo se creyó en el valor casi mágico de la existencia de los recursos de información. Se consideró central, procurar la mayor conectividad y el acceso sin exclusiones. Sin duda, no es la mera existencia de fuentes y las facilidades de encontrarlas, lo que permite transformar el vínculo de la sociedad con la información, sino la apropiación de esos recursos.

Es de justicia reconocer el trabajo de investigación y normalización que se ha desarrollado abordando temas asociados a la apropiación y el manejo inteligente como: la búsqueda de algoritmos, la arquitectura de información, los diseños ergonómicos, la calidad de las interfaces o los procesos cognitivos. En forma creciente los gobiernos y los sistemas educativos comprenden la significación y el impacto de estos recursos. Han detectado la necesidad de incorporar en forma universal estas herramientas a la vida cotidiana y en paralelo, el valor de la interacción entre el manejo de fuentes y participación ciudadana.

Los usuarios de la información aprendieron a relacionarse con la información a través de los buscadores, usando los vínculos hipertextuales, navegando entre distintas estructuras. Ese vínculo condicionó fuertemente sus hábitos y sobre todo, sus expectativas con respecto a los recursos de información que le son presentados. 
Aquel OPAC que en su camino había ingresado a la Web, presenta, pese a los esfuerzos de las interfaces desarrolladas, muchas deficiencias para estar en paridad con este nuevo universo. Los usuarios de información están condicionados por las pautas de operación de la red, las han internalizado. El OPAC recién llegado a la Web se presenta como rígido, plano, poco dialógico.

Las fuentes de información en Internet son muchas, se multiplican, y los esfuerzos por optimizar la calidad de la operación, la amigabilidad y la usabilidad, también. Los usuarios creen y sienten que no dependen de un OPAC para encontrar información. Y si tienen que elegir entre una fuente de calidad y solidez pero inabordable y una menos rigurosa pero amigable, eligen aquella con la que pueden mantener un vínculo simple, claro y cómodo.

En ese sentido sostiene Byrum": "Nuestras bibliografías nacionales y catálogos de bibliotecas son el resultado de siglos de esfuerzo intelectual y capital real. Teniendo en cuenta las grandes inversiones realizadas para crear y mantener tales interfaces a sus colecciones, las bibliotecas de cualquier parte deben buscar las oportunidades para dar las prestaciones necesarias con el fin de atraer a los usuarios para que sigan utilizando los OPACs como mecanismos valiosos de acceso..."

$Y$ concluye: "Ha llegado el momento de una actuación inmediata, si no queremos que nuestros usuarios abandonen no sólo los recursos que hemos recogido para ellos sino también las herramientas que les hacen estar disponibles"

En el marco de estos desafíos, y concientes de que no se trata de renunciar a la calidad y el rigor, los OPACS comienzan a alterar su presentación. Así:

- Incorporan hipertextualidad;

- optimizan los cuadros de diálogo;

- modifican los formatos de salida;

- incluyen imágenes;

- incorporan sumarios y textos completos asociados al registro.

Y por sobre todas las cosas, se repiensan y empiezan a incorporar la visión del beta perpetuo. ${ }^{2}$

Esto es, a pensarse en constante crecimiento y transformación sin por eso renunciar a la descripción sólida y rigurosa que les dio origen.

\footnotetext{
${ }^{1}$ Byrum, J. D. Jr. (2005). Recomendaciones para la mejora urgente que necesita el OPAC y el papel de la Agencia Bibliográfica Nacional para llevarla a cabo. Extraído el 21 de febrero de 2013 de: http://archive.ifla.org/IV/ifla71/papers/124s_trans-Byrum.pdf. Ponencia presentada a la 71era Conferencia General de IFLA, efectuada en la ciudad de Oslo, Suecia del 14 al 18 de agosto de 2005.

${ }^{2}$ Término acuñado por Tim O'Reilly (2005), que expresa loa convicción de que una estructura Web, está en perfecto perfeccionamiento y crecimiento por lo que no puede considerarse nunca consolidada.
} 


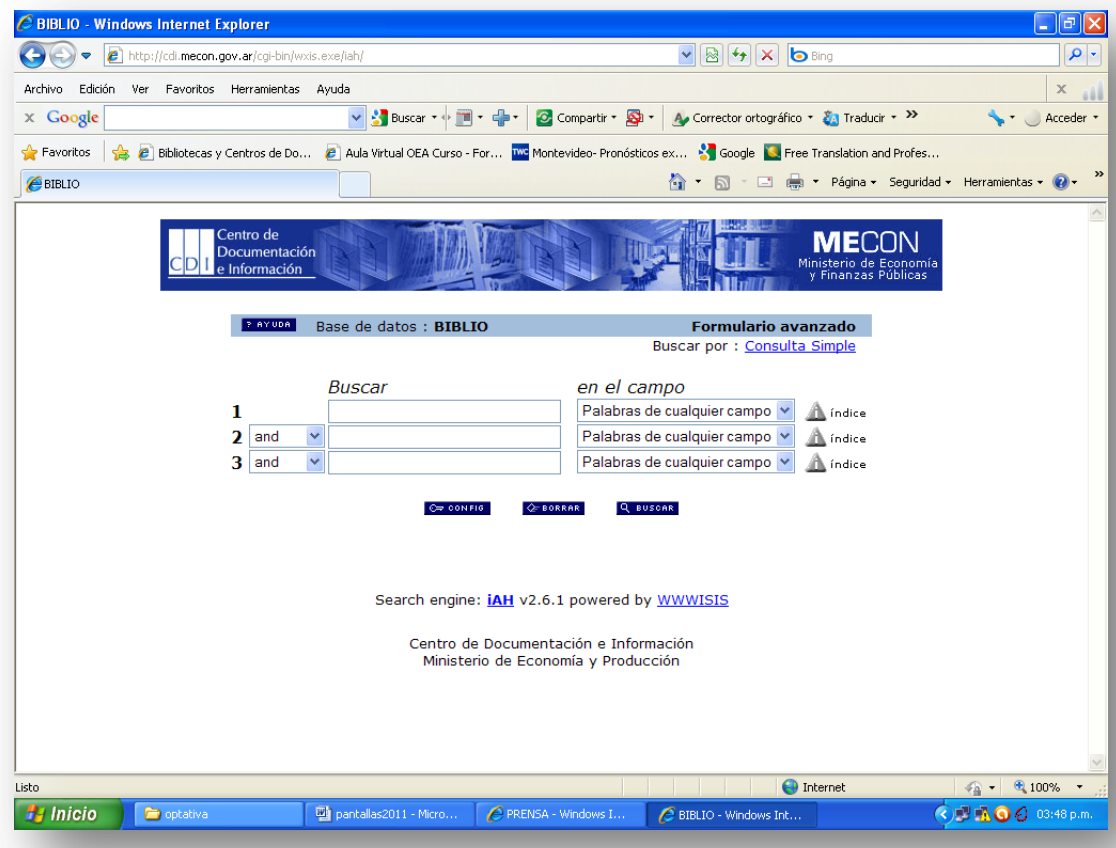

Figura 4. La interfaz modifica presentación buscando ser simples, claros y amigables.

En suma, los marcos Web, han aportado significativamente al crecimiento y la evolución del OPAC. Han puesto al usuario más allá de los límites físicos de la biblioteca. Y contribuido a consolidar un vínculo de diferente naturaleza con la información y sus fuentes.

Los llamados catálogos de tercera generación, se proponen superar las deficiencias de comunicación y adecuación de los llamados catálogos de segunda generación, se centran en optimizar la interfaz y asistir al usuario.

\section{LA WEB COMO PLATAFORMA, LA WEB 2.0 EL OPAC 2.0}

\section{La Web 2.0}

Como parte de la evolución del espacio $W e b$, se incorporan un conjunto de prestaciones y utilidades que comparten una misma filosofía. A la tan deseada interacción con el usuario, se suma la búsqueda de la horizontalidad, la participación y el pensamiento colectivo.

No se trata de aplicaciones o herramientas nuevas en sí mismas. La innovación reside en la conjunción. Los dispositivos, convergen se potencian e interactúan.

Un nuevo espacio Web se estructura para crear información y construir pensamiento en un entorno colaborativo. Este ámbito que propicia la inteligencia colectiva, es denominado: Web 2.0, en alusión a una nueva generación Web. El término fue utilizado por primera vez en el año 2004 por Tim O’Really. 
Como vimos, la singularidad no reside en los desarrollos, aplicaciones o programas, que se incorporan sino en la filosofía que subyace en la conjunción de esos desarrollos.

¿Qué aspectos definen estos espacios?

- Utilizan la estructura Web como plataforma;

- no se sustentan en una normativa, una herramienta o un método, sino en la conjunción de recursos orientados a la construcción de un espacio;

- en las opciones de su diseño, se expresa la vocación de la comunicación (arquitectura de la participación);

- no buscan la hegemonía de los creadores/responsables de los sitios;

- sin abdicar de su misión, se centran en la coordinación, no en el control;

- se orientan a un universo colaborativo y auspician y propician un comunidad de usuarios;

- conciben el conocimiento como una creación dinámica y colectiva, basada en la cooperación y el crecimiento dialéctico;

- presuponen organizaciones flexibles que por flexibles no son, ni se consideran menos sólidas;

- establecen un nuevo vínculos con los usuarios, de los usuarios con los contenidos y de los usuarios entre sí;

- incluyen el concepto de "beta perpetuo" asociado al diálogo, al crecimiento y a la mejora constante;

- no son solamente espacios lógicos en la Web sino y antes que nada, una forma de pensar, una actitud que puede empapar las acciones presenciales.

Listemos algunas de las tecnologías de la Web 2.0:

- Redes sociales de alcance general como Facebook.

- Mensajería instantánea.

- RSS.

- Etiquetado.

- Blogs.

- Wikis.

- Mashups.

Y otras tecnologías aún no incorporadas, o aún no creadas que se sumarán a las existentes o las sustituirán.

\section{La Biblioteca 2.0}

Lo usuarios de la información, que se desempeñan en el universo 2.0 donde prima la participación y la horizontalidad, esperan una Biblioteca 2.0 y un OPAC 2.0

La biblioteca 2.0 utiliza los recursos, la actitud y la filosofía de la Web 2.0.

El Documentalista Enredado ${ }^{1}$, enuncia las características básicas de la Biblioteca 2.0:

\footnotetext{
${ }^{1}$ El Documentalista Enredado (2010) ¿Qué hay de Web 2.0en la Biblioteca 2.0? El Documentalista Enredado: Biblioteconomía, Infonomía, Internet y Nuevas Tecnologías. Extraído el 21 de febrero de 2013 de: http://www.documentalistaenredado.net/918/que-hay-de-web-2-0-en-la-biblioteca-2-0/ 
1. Debe ser abierta para permitir el desarrollo y mejora de sus servicios y funcionamiento.

2. Debe ser interactiva de tal manera que sus usuarios puedan contribuir e interactuar con las herramientas disponibles en la Web 2.0.

3. Debe ser convergente para que las distintas herramientas de la Web 2.0 le permitan cumplir sus objetivos.

4. Debe ser colaborativa de tal forma que los usuarios y los bibliotecarios puedan comunicarse en el mismo nivel de autoridad.

5. Debe ser participativa puesto que la participación se halla en el cuadro central de la Web2.0, si no es participativa la Biblioteca 2.0 no tiene sentido."

6. Abierta, interactiva, convergente, colaborativa y participativa, la Biblioteca 2.0 cumplirá en un marco nuevo su vieja misión, renovando actitud desde un lugar diferente. Es muy posible que su liderazgo permanezca pero no sustentado en el control o la verticalidad, sino en su capacidad de construir espacios de pensamiento cada vez más convergentes, integrados y democráticos.

\section{EI OPAC 2.0}

Un OPAC 2.0 es el OPAC que incorpora las herramientas y la filosofía de la Web 2.0 y la Biblioteca 2.0.

Las interfaces que una vez propiciaron la comunicación del usuario con la estructura, se avocan a dar valor agregado a los recursos, hacer aportes que enriquecen los contenidos de información y permitir una nueva comunicación.

Sin perder el rigor de la descripción, el OPAC se convierte también en un sitio para consultas, opiniones, difusión y comentarios. Una suma de acciones bidireccionales expresan las modificaciones del vínculo de los usuarios con la información.

Sostiene Dídac Margaix-Arnal:

“...se pueden marcar cuatro objetivos del OPAC 2.0:

En primer lugar las tecnologías 2.0 son una más de las piezas que ayudan a mejorar la experiencia del usuario (junto a otras como la Usabilidad, la Arquitectura de la Información, etc.).

En segundo lugar si sus datos son más abiertos el OPAC puede posicionarse mejor entre las herramientas de recuperación de información utilizadas por los usuarios.

En tercer lugar el uso de información social y el aprovechamiento de la inteligencia colectiva ayudarán a reforzar el papel del OPAC como una herramienta de descubrimiento de información y no sólo como localizador de documentos ya conocidos.

Ahora bien, para que el usuario aporte información al sistema es necesario el cuarto objetivo: que el OPAC sea una herramienta útil y que la información que aporte el usuario revierta en un beneficio directo para sus propios objetivos." 
Veamos qué funciones permite la incorporación de las herramienta del 2.0 que diferencian al OPAC y le dan valor agregado:

- Seleccionar documentos;

- difundir los documentos;

- reunir documentos en directorios;

- señalar documentos como favoritos;

- hacer comentarios y compartirlos;

- sugerir adquisiciones de documentos;

- sugerir enlaces o fuentes vinculadas;

- sugerir la incorporación de datos de la descripción a los registros;

- enriquecer los registro bibliográficos con, por ejemplo: imágenes del documento como la cubierta o la portada, críticas, biografías, reportajes o tablas de contenido;

- incorporar herramientas de redes sociales;

- permitir la suscripción a canales RSS;

- permitir el intercambio entre usuarios de valoraciones, opiniones y comentarios;

- enlazar al OPAC con otras herramientas del entorno 2.0. 


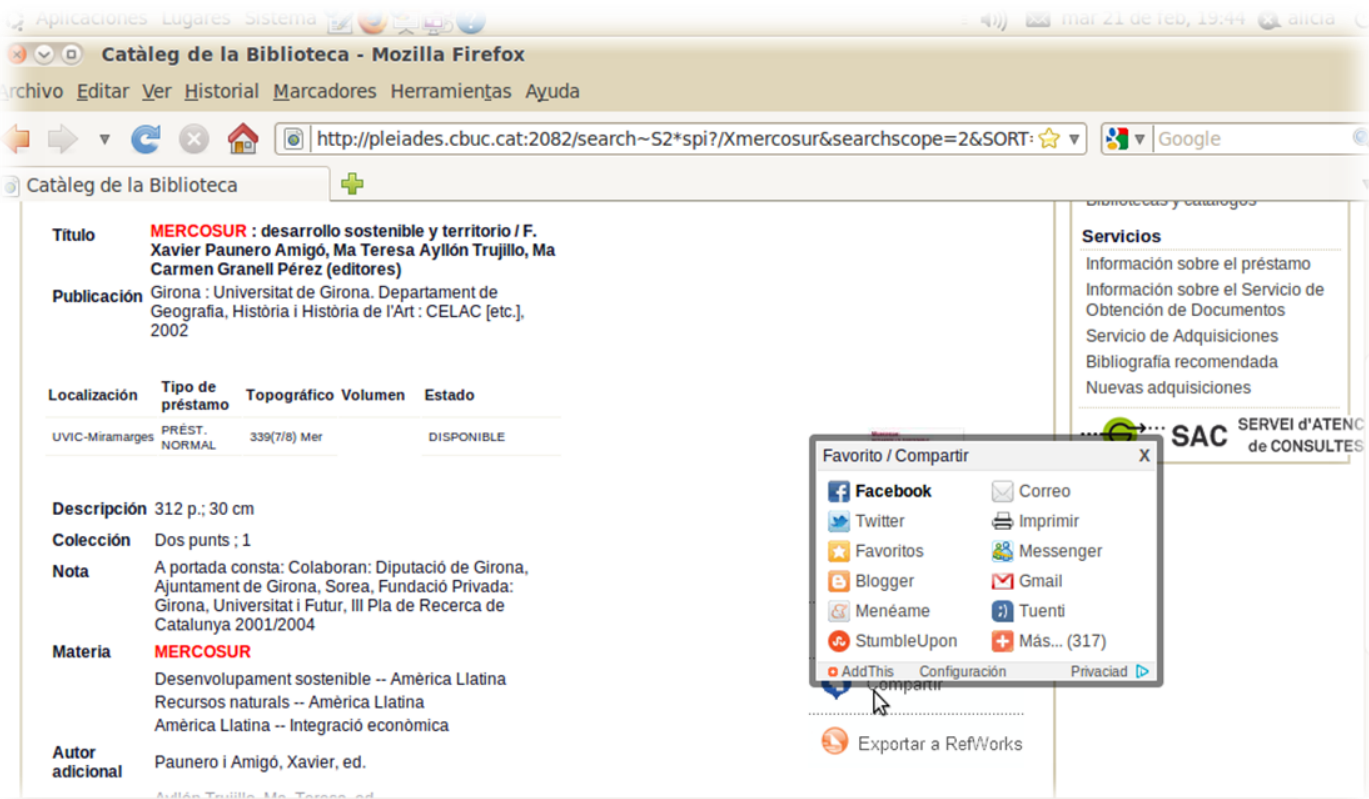

Figura 5. Resultados de búsqueda en el OPAC de la Biblioteca de la Universidad del Vic, (España) permite compartir los resultados con aportes de los usuarios.

Todas estas funciones, sumadas al perfeccionamiento que las interfaces ya habían desarrollado en términos de personalización de búsquedas y gestión de resultados y los avances en amigabilidad, y usabilidad construyen un nuevo catálogo. Se trata de un catálogo que responde a un nuevo vínculo de los usuarios con la información. Ese parece ser el signo del camino del OPAC.

En esta ocasión se evidencia singularmente una situación que bien pudo ser preexistente:

El OPAC que cambia para acompañar un momento del pensamiento colectivo, se reformula y crece acompañando ese pensamiento. El impacto de esa conversión, incide en toda la práctica organizacional.

Ya no se trata sólo de cambiar para seguir siendo una oferta a considerar y permanecer. 


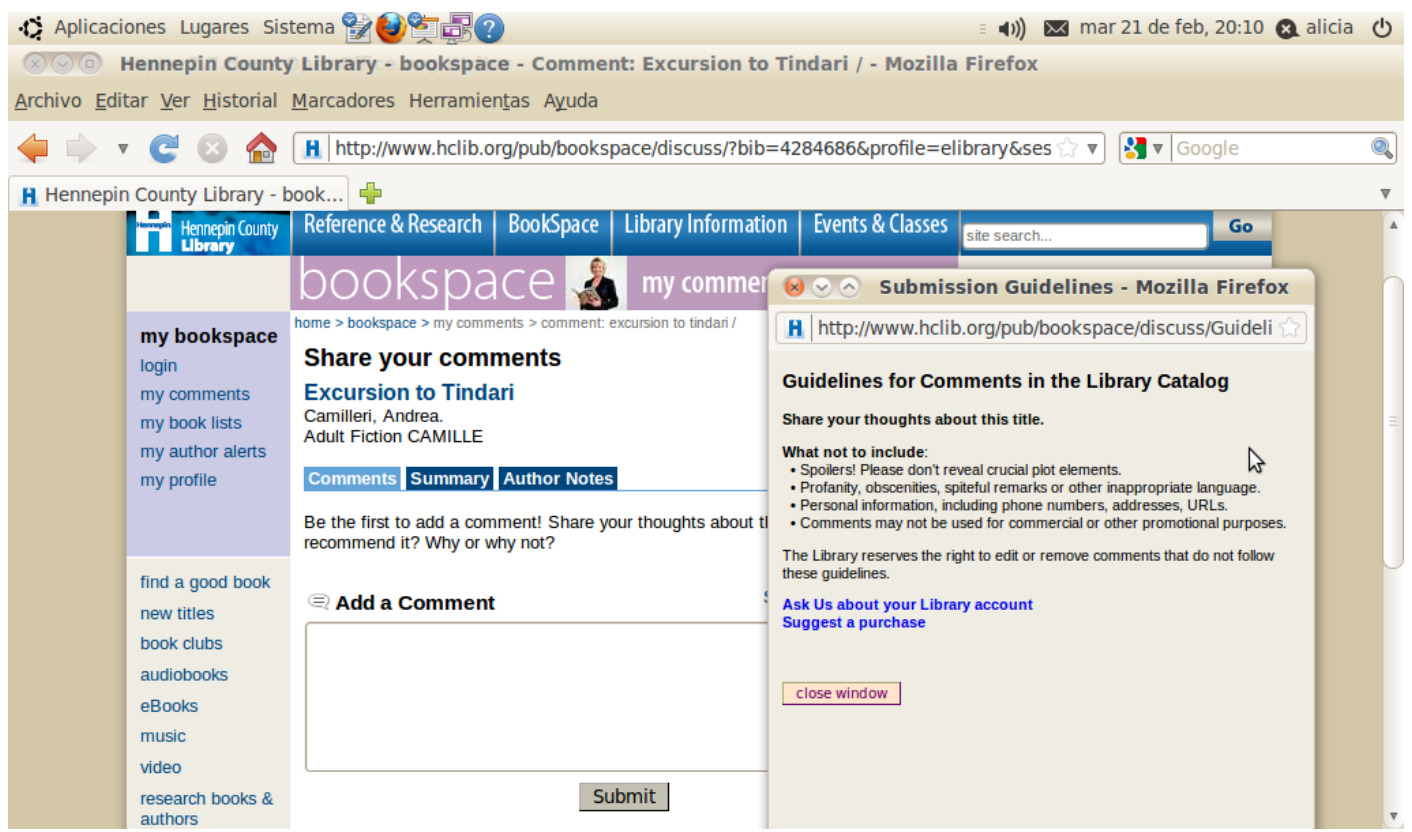

Figura 6. El OPAC de la Biblioteca del Condado de Hennepin, (Estados Unidos) permite que los usuarios aporten comentarios a un registro y los compartan, a la derecha se puede ver desplegado una ventana con directrices para comentar el catálogo.

\section{CONCLUSIÓN}

Estamos en presencia de una trasformación que hace a la actitud y a la comprensión del vínculo de los usuarios con la información. Se trata de la visión de la información como derecho y el concepto de conocimiento como construcción colectiva en permanente crecimiento gracias a la interacción.

Esta actitud involucra a los profesionales de la información, a las organizaciones que construyen y difunden conocimiento, a los gobiernos y esencialmente al colectivo social.

No se trata de un cambio de apariencia, recursos gráficos o espacios para recibir opiniones, sino de una visión que no asocie calidad o rigor a control y directividad.

¿Podremos dejar atrás la dicotomía entre "los que saben" y "Ios que no saben"?

Sostiene David Maniega': "...cuando se trabaja en un catálogo se piensa en sus procesos internos, y no en el usuario y sus intereses, pero claro, esto choca con la finalidad del mismo, ofrecer servicio a éste último... Si bien es cierto que pensamos en crear grandes catálogos con todos nuestros fondos para que el usuario encuentre nuestros documentos y recursos, no estamos pensando en ellos, y tampoco "para" ellos..."

¿Podremos repensar nuestras acciones, dejar de ver "nuestros" catálogos como "nuestros" productos y repensar las prácticas sin desdecirnos de nuestra misión?

${ }^{1}$ Maniega, David (2008). “Opac 2.0: el futuro dentro de una realidad tangible”. Anuario ThinkEPI, 2008, pp. 41-45. Extraído el 21 de febrero de 2013 de: http://www.thinkepi.net/opac-20-el-futuro-dentro-de-una-realidad-tangible Cuadernos de Documentación Multimedia Vol. 24. 
¿Podremos aportar una visión verdaderamente democratizadora a nuestras acciones?

¿Seremos capaces de "leer" el fuerte mensaje que podemos dar a la sociedad sobre el pensar en paridad?

Llevamos varias décadas promoviendo la paridad lógica que sustentan los protocolos de Internet y el acceso universal. Ya es hora de promover otras paridades desde nuestras prácticas, paridades que crean ciudadanía y sustentan democracia a partir de la participación.

\section{BIBLIOGRAFÍA CITADA}

1 Hildreth, Charles R. Pursuing the ideal: generations of online catalogs. En: Aveney, B. y B. Butler, eds. Online catalogs, online reference: converging trends. Chicago: ALA. p. 31-56.

2 Marcos Mora, Mari-Carmen (2004). Pautas para el diseño y la evaluación de interfaces de usuario. En: Rovira, Cristòfol; Codina, Lluís; Marcos, Mari-Carmen; Palma, María del Valle. Información y documentación digital. Barcelona: IULA; Documenta Universitaria, 2004. ISBN 84-96367-09-6.

3 Rodriguez Yunta, Luis; Giménez Toledo, Elea (2004). Lo que los usuarios piensan de las bases de datos bibliográficas y no se atreven a decir. ¿Es posible un diseño centrado en el usuario? Preprint de la primera versión de la Comunicación enviada a Infogestión. IX Jornadas Españolas de Documentación. Fesabid 14-15 abril 2005 Preprint entregado el 3 de noviembre de 2004

4 Fingerhut, Michel (2000). Le site Web de la bibliothèque considéré comme un espace. Bulletin des Bibliothéques de France, 45 (3), 78 - 82. Extraído el 21 de febrero de 2013: http://bbf.enssib.fr/consulter/10-finger.pdf

5 Játiva Miralles, Victoria (2002) Opac-portal: una nueva forma de ofrecer los recursos y servicios de la biblioteca. El Profesional de la Información, 11 (6), 442-453.

6 Saorín Pérez, Tomás (2006). Modelo conceptual para la automatización de bibliotecas en el contexto digital. Universidad de Murcia. [Tesis doctoral dirigida por José Vicente Rodríguez Muñoz].

7 González Fernández-Villavicencio, Nieves (2009). La biblioteca expandida en código abierto. Boletín de la Asociación Andaluza de Bibliotecarios, 24, (96-97).

8 Byrum, J. D. Jr. (2005). Recomendaciones para la mejora urgente que necesita el OPAC y el papel de la Agencia Bibliográfica Nacional para llevarla a cabo. Extraído el 21 de febrero de 2013 de: http://archive.ifla.org/IV/ifla71/papers/124s trans-Byrum.pdf. Ponencia presentada a la 71era Conferencia General de IFLA, efectuada en la ciudad de Oslo, Suecia del 14 al 18 de agosto de 2005.

9 O'Reilly, Tom (2005) What Is Web 2.0. Design Patterns and Business Models for the Next Generation of Software. Extraído el 21 de febrero de 2013 de: http://oreilly.com/web2/archive/what-is-web-20.html

10 El Documentalista Enredado (2010) ¿Qué hay de Web 2.0 en la Biblioteca 2.0? El Documentalista Enredado: Biblioteconomía, Infonomía, Internet y Nuevas Tecnologías. Extraído el 21 de febrero de 2013 de: http://www.documentalistaenredado.net/918/que-hay-de-web-2-0-en-la-biblioteca-2-0/ 
11 Maniega, David (2008). "Opac 2.0: el futuro dentro de una realidad tangible”. Anuario ThinkEPI, 2008, pp. 41-45. Extraído el 21 de febrero de 2013 de: http://www.thinkepi.net/opac-20-el-futuro-dentro-de-unarealidad-tangible

\section{BIBLIOGRAFÍA CONSULTADA}

Álvarez Marañón, Gonzalo (2010) Amenazas 2.0 para la Biblioteca 2.0, V Congreso Nacional de Bibliotecas Públicas, Gijón, España 2010: Extraído el 21 de febrero de 2013 de: http://travesia.mcu.es/portalnb/jspui/bitstream/10421/4975/1/GonzaloAlvarez.pdf

Duchemin, Pierre-Yves (2005). L'enrichissement des catalogues ? Et après ? Bulletin des Bibliothéques de France, 50 (4), 21-27. Extraído el 21 de febrero de 2013 de: http://bbf.enssib.fr/sdx/BBF/pdf/bbf-20054/bbf-2005-04-0021-004.pdf

Frías, J. A. (1996). Los registros bibliográficos y las necesidades informativas de los usuarios. Scire: representación y organización del conocimiento, 1 (2), 23-50.

Gómez Hernández, José A.; Saorín Pérez, Tomás (2006) Alfabetizarse desde dentro en la Web2.0: Aprender a informarse y comunicarse en redes sociales. Extraído el 21 de febrero de 2013 de: http://gredos.usal.es/ispui/bitstream/10366/119290/1/EB18 N156 P131-137.pdf

Hassan, Yusef; Martín Fernández, Francisco J.; lazza, Ghzala (2004) Diseño web Centrado en el Usuario: Usabilidad y Arquitectura de la Información. Hipertext.net, 2. Extraído el 21 de febrero de 2013 de: http://eprints.rclis.org/8998/

Hildreth, Charles. R. (1988). Online library catalogues as information retrieval systems: what can we learn from research? En Yates-Merger, P. A., ed. Future trends in information science and technology. London: Taylor Graham. 9-25.

Hildreth, Charles. R.(1989). General introduction; OPAC research: laying the groundwork for future OPAC design. En: Hildreth, Charles. R., ed. The online catalogue: developments and directions. London: Library Association. p. 1-24.

IFLA (2003). Guidelines for online public access catalogue (OPAC) displays. Extraído el 21 de febrero de 2013 de: http://www.ifla.org/VII/s13/guide/opacguide03.htm

Lamarca Lapuente, María Jesús (2013) Del catálogo tradicional al catálogo en línea. En: Hipertexto: El nuevo concepto de documento en la cultura de la imagen. Extraído el 21 de febrero de 2013 de: http://www.hipertexto.info/documentos/catalogo.htm

Játiva Miralles, Victoria (2009) El catálogo: un recurso en expansión. Anales de Documentación, Nro. 12, 6991. Extraído el 21 de febrero de 2013 de: digitum.um.es/jspui/bitstream/10201/9652/1/p.\%2069-91.pdf

Maniega, David (2008). "Opac 2.0: el futuro dentro de una realidad tangible". Anuario ThinkEPI, 2008, pp. 41-45. Extraído el 21 de febrero de 2013 de: http://www.thinkepi.net/opac-20-el-futuro-dentro-de-unarealidad-tangible 
Margaix-Arnal, Dídac. (2007). El Opac 2.0: las tecnologías de la Web 2.0 aplicadas a los catálogos bibliográficos. Actas VI Workshop Calsi. 2007. Extraído el 21 de febrero de 2013 de: http://www.calsi.org/2007/wp-content/uploads/2007/11/didac margaix.pdf

Margaix-Arnal, Dídac. (2008). El OPAC 2.0 : Puerta de Acceso a los Contenidos de la Biblioteca. En: IV Congreso Nacional de Bibliotecas Públicas, La Coruña (España), 24-26 de setiembre de 2008. Extraído el 21 de febrero de 2013 de : http://eprints.rclis.org/bitstream/10760/3802/1/Congreso BP Margaix ELIS.pdf

Ortega, Marisa (2009) Un usuario desconcertado es un usuario perdido: funcionalidades del OPAC 2.0. Extraído el 21 de febrero de 2013 de: http://eprints.rclis.org/bitstream/10760/14252/1/opac 2.0.pdf

Rodríguez Yunta, Luis; Giménez Toledo, Elea (2004). Lo que los usuarios piensan de las bases de datos bibliográficas y no se atreven a decir. ¿Es posible un diseño centrado en el usuario? Preprint de la primera versión de la Comunicación enviada a Infogestión. IX Jornadas Españolas de Documentación. Fesabid 14-15 abril 2005. Preprint entregado el 3 de noviembre de 2004.

Tramullas, Jesús (2011). El emperador va desnudo... y el OPAC también. En: tramullas.com. Extraído el 21 de febrero de 2013 de: http://tramullas.com/2011/02/17/el-emperador-va-desnudo-y-el-opac-tambien/

Yee, M. M. (1999, Agosto). Directrices para las visualizaciones en catálogos en línea. Ponencia presentada a 65th IFLA Council and General Conference. Bangkok, Thailandia. Extraído el 21 de febrero de 2013 de: http://archive.ifla.org/IV/ifla65/papers/098-131s.htm

Yu, H. \& Young, M. (2004) The Impact of Web Search Engines on Subject Searching in OPAC. Information Technology and Libraries, 23 (4), 168-180. 